\title{
Coumarin-4-ylmethoxycarbonyls as Phototriggers for Alcohols and Phenols
}

\section{Akinobu Z. Suzuki, Takayoshi Watanabe, Mika Kawamoto, Keiko \\ Nishiyama, Hirotaka Yamashita, Michiko Iwamura and Toshiaki Furuta*}

Department of Biomolecular Science, Toho University, 2-2-1 Miyama, Funabashi, 274-8510

Japan

furuta@biomol.sci.toho-u.ac.jp

\section{Synthesis}

1,2,3,4-Di-O-isopropylidene-D-galactopyranosyl 7-methoxycoumarin-4-ylmethoxycarbonate (1a).

To a stirred solution of $82.9 \mathrm{mg}(0.318 \mathrm{mmol})$ of 1,2,3,4-di-O-isopropylidene-D-galactopyranose in $\mathrm{CH}_{2} \mathrm{Cl}_{2}(2 \mathrm{~mL})$ and $\mathrm{CH}_{3} \mathrm{CN}$ (2.5 mL) was added $49.3 \mathrm{mg}(0.404 \mathrm{mmol})$ of 4-dimethylaminopyridine and $104.0 \mathrm{mg}(0.387 \mathrm{mmol})$ of 7-methoxycoumarin-4ylmethoxycarbonyl chloride. The reaction mixture was stirred at room temperature for 45 min. The solvents were removed with rotovap. Purification by flash column chromatography (15 g of $\left.\mathrm{SiO}_{2}, \mathrm{CH}_{2} \mathrm{Cl}_{2} / \mathrm{CH}_{3} \mathrm{OH}=25 / 1\right)$ gave $123.9 \mathrm{mg}(0.237 \mathrm{mmol}$. $94 \%$ yield) of 1a.

1a. ${ }^{1} \mathrm{H} \mathrm{NMR}\left(\mathrm{CDCl}_{3}\right) \delta 1.34(3 \mathrm{H}, \mathrm{s}), 1.35(3 \mathrm{H}, \mathrm{s}), 1.46(3 \mathrm{H}, \mathrm{s}), 1.52(3 \mathrm{H}, \mathrm{s}), 3.88(3 \mathrm{H}, \mathrm{s}), 4.09(1 \mathrm{H}, \mathrm{ddd}, \mathrm{J}=1.6,5.5 \& 8.0 \mathrm{~Hz})$, $4.26(1 \mathrm{H}, \mathrm{dd}, \mathrm{J}=1.6 \& 7.8 \mathrm{~Hz}), 4.31-4.43(3 \mathrm{H}, \mathrm{m}), 4.64(1 \mathrm{H}, \mathrm{dd}, \mathrm{J}=2.4 \& 7.8 \mathrm{~Hz}), 5.27(1 \mathrm{H}, \mathrm{d}, \mathrm{J}=14 \mathrm{~Hz}), 5.34(1 \mathrm{H}, \mathrm{d}, \mathrm{J}=14 \mathrm{~Hz})$, $5.55(1 \mathrm{H}, \mathrm{d}, \mathrm{J}=4.9 \mathrm{~Hz}), 6.39(1 \mathrm{H}, \mathrm{s}), 6.85(1 \mathrm{H}, \mathrm{s}), 6.87(1 \mathrm{H}, \mathrm{d}, \mathrm{J}=8.4 \mathrm{~Hz}), 7.41(1 \mathrm{H}, \mathrm{d}, \mathrm{J}=8.4 \mathrm{~Hz}) ;{ }^{13} \mathrm{C} \mathrm{NMR}\left(\mathrm{CDCl}{ }_{3} / \mathrm{TMS}\right) \delta$ $24.49,24.91,25.95,26.03,55.80,64.53,65.67,67.32,70.37,70.68,70.82,96.25,101.23,108.88,109.79,110.33,110.44$, 112.67, 124.43, 148.37, 154.48, 155.58, 160.71, 162.90; UV $\lambda_{\max } / n m(\varepsilon)$ solvent A: $322(12,100)$, solvent C: 323 (13,100).

1,2,3,4-Di-O-isopropylidene-D-galactopyranosyl 6-bromo-7-hydroxycoumarin-4-ylmethoxycarbonate (1b). 
To a stirred solution of $325.8 \mathrm{mg}(1.202 \mathrm{mmol})$ of 6-bromo-7-hydroxy-4-hydroxymethylcoumarin $(\mathbf{2 b})$ in $\mathrm{CH}_{3} \mathrm{CN}(5 \mathrm{~mL})$ was added $353.2 \mathrm{mg}(2.89 \mathrm{mmol})$ of 4-dimethylaminopyridine and $264.0 \mathrm{mg}(1.31 \mathrm{mmol})$ of 4-nitrophenyl chloroformate. The reaction mixture was stirred for $6 \mathrm{hr}$ at room temperature. Then, another $152.5 \mathrm{mg}(1.25 \mathrm{mmol})$ of 4-dimethylaminopyridine and $515 \mathrm{mg}$ (1.99 mmol) of 1,2,3,4-di-O-isopropylidene-D-galactopyranose were added to the reaction mixture. After $24 \mathrm{hr}$, the mixture was diluted with $\mathrm{CHCl}_{3}$, washed with $0.5 \mathrm{M}$ citric acid and sat. $\mathrm{NaCl}$, and dried over $\mathrm{MgSO}_{4}$. Purification by column chromatography $\left(50 \mathrm{~g}\right.$ of $\left.\mathrm{SiO}_{2}, \mathrm{n}-\mathrm{Hexane} / \mathrm{EtOAc}=3 / 2\right)$ gave $221.9 \mathrm{mg}(0.398 \mathrm{mmol} .33 \%$ yield $)$ of $\mathbf{1 b}$.

1b. ${ }^{1} \mathrm{H}$ NMR (CDCl $\left.3 / \mathrm{TMS}\right) \delta 1.34(3 \mathrm{H}, \mathrm{s}), 1.35(3 \mathrm{H}, \mathrm{s}), 1.47(3 \mathrm{H}, \mathrm{s}), 1.53(3 \mathrm{H}, \mathrm{s}), 4.08-4.13(2 \mathrm{H}, \mathrm{m}), 4.25(1 \mathrm{H}, \mathrm{dd}, \mathrm{J}=1.5,7.8$ Hz), 4.33-4.37 (3H, m), $4.63(1 \mathrm{H}, \mathrm{dd}, \mathrm{J}=2.3,7.8 \mathrm{~Hz}), 5.24(1 \mathrm{H}, \mathrm{d}, \mathrm{J}=14 \mathrm{~Hz}), 5.27(1 \mathrm{H}, \mathrm{d}, \mathrm{J}=14 \mathrm{~Hz}), 5.55(1 \mathrm{H}, \mathrm{d}, \mathrm{J}=5 \mathrm{~Hz}), 6.41$ $(1 \mathrm{H}, \mathrm{s}), 7.04(1 \mathrm{H}, \mathrm{s}), 7.62(1 \mathrm{H}, \mathrm{s}) ;{ }^{13} \mathrm{C} \mathrm{NMR}\left(\mathrm{CDCl}_{3} / \mathrm{TMS}\right) \delta 24.45,24.86,25.90,25.99,64.19,65.67,67.43,70.32,70.64,70.78$, $96.21,104.37,106.86,108.91,109.80,111.24,111.72,126.84,147.57,154.36,154.41,155.83,160.29 ;$ UV $\lambda_{\max } / n m(\mathcal{E})$ solvent A: $374(15,000)$, solvent B: $376(14,000)$.

\section{1,2,3,4-Di-O-isopropylidene-D-galactopyranosyl 7-diethylaminocoumarin-4-ylmethoxycarbonate (1c).}

To a stirred suspension of $271.6 \mathrm{mg}(1.04 \mathrm{mmol})$ of 7-diethylamino-4-hydroxymethylcoumarin in $\mathrm{CH}_{2} \mathrm{Cl}_{2}(7 \mathrm{~mL})$ was added 141.7 $\mathrm{mg}(1.16 \mathrm{mmol})$ of 4-dimethylaminopyridine and $232.0 \mathrm{mg}(1.15 \mathrm{mmol})$ of 4-nitrophenyl chloroformate. The reaction mixture was stirred for $10 \mathrm{hr}$ at room temperature. Thin layer chromatography indicated that the formation of intermediate 4-nitrophenyl carbonate completed. Then, another $142.2 \mathrm{mg}(1.16 \mathrm{mmol})$ of 4-dimethylaminopyridine and $265.9 \mathrm{mg}(1.02 \mathrm{mmol})$ of 1,2,3,4-di$\mathrm{O}$-isopropylidene-D-galactopyranose were added to the reaction mixture. After $20 \mathrm{hr}$, the mixture was diluted with $\mathrm{CH}_{2} \mathrm{Cl}_{2}$, washed with sat. $\mathrm{NaHCO}_{3}$ and sat. $\mathrm{NaCl}$, and dried over $\mathrm{MgSO}_{4}$. Purification by flash column chromatography $\left(23 \mathrm{~g}\right.$ of $\mathrm{SiO}_{2}$, $\mathrm{CH}_{2} \mathrm{Cl}_{2} / \mathrm{CH}_{3} \mathrm{OH}=85 / 1$ then $\left.\mathrm{CH}_{2} \mathrm{Cl}_{2} / \mathrm{CH}_{3} \mathrm{OH}=50 / 1\right)$ gave $440.9 \mathrm{mg}(0.826 \mathrm{mmol} .81 \%$ yield $)$ of $1 \mathrm{c}$.

1c. ${ }^{1} \mathrm{H}$ NMR (CDCl 3 /TMS) $\delta 1.21(6 \mathrm{H}, \mathrm{t}, \mathrm{J}=7.3 \mathrm{~Hz}), 1.34(3 \mathrm{H}, \mathrm{s}), 1.35(3 \mathrm{H}, \mathrm{s}), 1.46(3 \mathrm{H}, \mathrm{s}), 1.52(3 \mathrm{H}, \mathrm{s}), 3.41(4 \mathrm{H}, \mathrm{q}, \mathrm{J}=7.3 \mathrm{~Hz})$, $4.08(1 \mathrm{H}, \mathrm{ddd}, \mathrm{J}=1.5,5 \& 8.5 \mathrm{~Hz}), 4.27(1 \mathrm{H}, \mathrm{dd}, \mathrm{J}=1.5,7.8 \mathrm{~Hz}), 4.33-4.37(3 \mathrm{H}, \mathrm{m}), 4.63(1 \mathrm{H}, \mathrm{dd}, \mathrm{J}=2.3,7.8 \mathrm{~Hz}), 5.23(1 \mathrm{H}, \mathrm{d}, \mathrm{J}=14$ Hz), $5.30(1 \mathrm{H}, \mathrm{d}, \mathrm{J}=14 \mathrm{~Hz}), 5.55(1 \mathrm{H}, \mathrm{d}, \mathrm{J}=4.9 \mathrm{~Hz}), 6.17(1 \mathrm{H}, \mathrm{s}), 6.50(1 \mathrm{H}, \mathrm{d}, \mathrm{J}=2.4 \mathrm{~Hz}), 6.57(1 \mathrm{H}, \mathrm{dd}, \mathrm{J}=2.4,8.9 \mathrm{~Hz}), 7.28(1 \mathrm{H}, \mathrm{d}$, $\mathrm{J}=8.9 \mathrm{~Hz}) ;{ }^{13} \mathrm{C} \mathrm{NMR}\left(\mathrm{CDCl}_{3} / \mathrm{TMS}\right) \delta 12.42(\mathrm{x} 2), 24.49,24.92,25.95,26.04,44.77(\mathrm{x} 2), 64.73,65.65,67.13,70.39,70.66,70.81$, $96.24,97.87,105.86,106.78,108.69,108.88,109.74,124.40,148.60,150.64,154.58,156.28,161.71 ;$ UV $\lambda_{\max } / n m(\varepsilon)$ solvent B: 
$250(11,400), 396(17,300)$.

\section{1,2,3,4-Di- $O$-isopropylidene-D-galactopyranosyl 6,7-dimethoxycoumarin-4-ylmethoxycarbonate (1d).}

To a stirred solution of $65.4 \mathrm{mg}(0.251 \mathrm{mmol})$ of 1,2,3,4-di-O-isopropylidene-D-galactopyranose in $\mathrm{CH}_{2} \mathrm{Cl}_{2}(5 \mathrm{~mL})$ and $\mathrm{CH}_{3} \mathrm{CN}(2$ $\mathrm{mL})$ was added $39.0(0.32 \mathrm{mmol})$ of 4-dimethylaminopyridine and $90.8 \mathrm{mg}(0.304 \mathrm{mmol})$ of 6,7-dimethoxycoumarin-4ylmethoxycarbonylchloride. The reaction mixture was stirred at room temperature for $2 \mathrm{hr}$. The solvent was removed with rotovap. Purification by flash column chromatography ( $20 \mathrm{~g}$ of $\mathrm{SiO}_{2}, \mathrm{CH}_{2} \mathrm{Cl}_{2} / \mathrm{CH}_{3} \mathrm{OH}=80 / 1$ then $\left.\mathrm{CH}_{2} \mathrm{Cl}_{2} / \mathrm{CH}_{3} \mathrm{OH}=50 / 1\right)$ gave $123.9 \mathrm{mg}$ (0.237 mmol. 94\% yield) of $\mathbf{1 d}$.

1d. ${ }^{1} \mathrm{H}$ NMR $\left(\mathrm{CDCl}_{3}\right) \delta 1.34(3 \mathrm{H}, \mathrm{s}), 1.35(3 \mathrm{H}, \mathrm{s}), 1.46(3 \mathrm{H}, \mathrm{s}), 1.52(3 \mathrm{H}, \mathrm{s}), 3.93(3 \mathrm{H}, \mathrm{s}), 3.95(3 \mathrm{H}, \mathrm{s}), 4.09(1 \mathrm{H}, \mathrm{ddd}, \mathrm{J}=1.5,5.5$ \& $8.0 \mathrm{~Hz}), 4.26(1 \mathrm{H}, \mathrm{dd}, \mathrm{J}=1.5 \& 7.8 \mathrm{~Hz}), 4.31-4.44(3 \mathrm{H}, \mathrm{m}), 4.64(1 \mathrm{H}, \mathrm{dd}, \mathrm{J}=2.4 \& 7.8 \mathrm{~Hz}), 5.28(1 \mathrm{H}, \mathrm{d}, \mathrm{J}=15 \mathrm{~Hz}), 5.34(1 \mathrm{H}, \mathrm{d}$, $\mathrm{J}=15 \mathrm{~Hz}), 5.54(1 \mathrm{H}, \mathrm{d}, \mathrm{J}=4.9 \mathrm{~Hz}), 6.43(1 \mathrm{H}, \mathrm{s}), 6.84(1 \mathrm{H}, \mathrm{s}), 6.87(1 \mathrm{H}, \mathrm{s}) ;{ }^{13} \mathrm{C} \mathrm{NMR}\left(\mathrm{CDCl}_{3} / \mathrm{TMS}\right) \delta 24.48,24.90,25.94,26.02$, $56.38,56.53,64.67,65.68,67.36,70.36,70.67,70.82,96.24,100.35,103.91,108.88,109.36,109.79,110.80,146.41,148.12$, 149.77, 153.01, 154.52, 160.89; UV $\lambda_{\max } / n m(\varepsilon)$ solvent A: $290(4,500), 344(10,800)$.

\section{1,2,3,4-Di- $O$-isopropylidene-D-galactopyranosyl 6-nitroveratryloxycarbonate (1e).}

To a stirred solution of $143.8 \mathrm{mg}(0.552 \mathrm{mmol})$ of 1,2,3,4-di-O-isopropylidene-D-galactopyranose in $\mathrm{CH}_{2} \mathrm{Cl}_{2}(3.5 \mathrm{~mL})$ and $\mathrm{CH}_{3} \mathrm{CN}$ $(1 \mathrm{~mL})$ was added $84.3 \mathrm{mg}(0.69 \mathrm{mmol})$ of 4-dimethylaminopyridine and $180.5 \mathrm{mg}(0.655 \mathrm{mmol})$ of 6-nitroveratryloxycarbonyl chloride. The reaction mixture was stirred at room temperature for $3 \mathrm{hr}$. The solvents were removed with rotovap. Purification by flash column chromatography $\left(20 \mathrm{~g}\right.$ of $\mathrm{SiO}_{2}, \mathrm{CH}_{2} \mathrm{Cl}_{2} / \mathrm{CH}_{3} \mathrm{OH}=98 / 1$ then $\left.\mathrm{CH}_{2} \mathrm{Cl}_{2} / \mathrm{CH}_{3} \mathrm{OH}=25 / 1\right)$ gave $169.1 \mathrm{mg}(0.339 \mathrm{mmol}$. $61 \%$ yield) of 1 .

1e. ${ }^{1} \mathrm{H}$ NMR $\left(\mathrm{CDCl}_{3}\right) \delta 1.32(3 \mathrm{H}, \mathrm{s}), 1.34(3 \mathrm{H}, \mathrm{s}), 1.46(6 \mathrm{H}, \mathrm{s}), 3.96(3 \mathrm{H}, \mathrm{s}), 4.01(3 \mathrm{H}, \mathrm{s}), 4.11(1 \mathrm{H}, \mathrm{ddd}, \mathrm{J}=1.0,5.5 \& 7.0 \mathrm{~Hz})$, $4.26(1 \mathrm{H}, \mathrm{dd}, \mathrm{J}=1.0 \& 8.0 \mathrm{~Hz}), 4.32-4.37(3 \mathrm{H}, \mathrm{m}), 4.62(1 \mathrm{H}, \mathrm{dd}, \mathrm{J}=2.2 \& 8.0 \mathrm{~Hz}), 5.53(1 \mathrm{H}, \mathrm{d}, \mathrm{J}=4.9 \mathrm{~Hz}), 5.59(1 \mathrm{H}, \mathrm{d}, \mathrm{J}=14 \mathrm{~Hz})$,

$5.63(1 \mathrm{H}, \mathrm{d}, \mathrm{J}=14 \mathrm{~Hz}), 7.09$ (1H, s), $7.74(1 \mathrm{H}, \mathrm{s}) ;{ }^{13} \mathrm{C} \mathrm{NMR}\left(\mathrm{CDCl}_{3} / \mathrm{TMS}\right) \delta 24.47,24.91,25.92$ (X 2), 56.40, 56.58, 65.71, 66.30, $67.10,70.35,70.67,70.88,96.25,108.09,108.82,109.47,109.75,127.12,139.41,148.16,153.81,154.67 ;$ UV $\lambda_{\max } / n m(\varepsilon)$ 
solvent A: $344(5,400)$.

\section{Phenyl 6-Bromo-7-hydroxycoumarin-4-ylmethoxycarbonate (3).}

To a stirred suspension of $134.9 \mathrm{mg}(0.498 \mathrm{mmol})$ of $\mathbf{2 b}$ in $\mathrm{CH}_{3} \mathrm{CN}(3.5 \mathrm{~mL})$ was added $147 \mu \mathrm{L}(0.84 \mathrm{mmol})$ of diisopropyl ethylamine and $63 \mu \mathrm{L}(0.50 \mathrm{mmol})$ of phenyl chloroformate. The reaction mixture was stirred at room temperature for $3.5 \mathrm{hr}$. The solvents were removed with rotovap. Purification by flash column chromatography $\left(17 \mathrm{~g}\right.$ of $\left.\mathrm{SiO}_{2}, \mathrm{n}-\mathrm{Hexane} / \mathrm{EtOAc}=5 / 2\right)$ gave $117.7 \mathrm{mg}(0.301 \mathrm{mmol} .60 \%$ yield $)$ of $\mathbf{3}$.

3. ${ }^{1} \mathrm{H}$ NMR ( $\left.\mathrm{CDCl}_{3} / \mathrm{TMS}\right) \delta 5.37(2 \mathrm{H}, \mathrm{s}), 6.49(1 \mathrm{H}, \mathrm{s}), 7.05(1 \mathrm{H}, \mathrm{s}), 7.10-7.15(3 \mathrm{H}, \mathrm{m}), 7.40-7.45(2 \mathrm{H}, \mathrm{m}), 7.67(1 \mathrm{H}, \mathrm{s}) ;{ }^{13} \mathrm{C} \mathrm{NMR}$ $\left(\mathrm{CDCl}_{3} / \mathrm{TMS}\right) \delta 65.28,103.20,106.32,109.90,110.35,121.22,126.40,128.74,129.69,148.60,150369,152.43,153.89,157.59$, 159.52; UV $\lambda_{\max } / n m(\varepsilon)$ solvent A: $372(17,900)$.

\section{6-Bromo-7-methoxymethoxy-4-hydroxymethylcoumarin (4).}

To a stirred suspension of $1.0846 \mathrm{~g}(4.001 \mathrm{mmol})$ of $\mathbf{2 b}$ in $\mathrm{CH}_{2} \mathrm{Cl}_{2}(10 \mathrm{~mL})$ was added $836 \mu \mathrm{L}$ (4.80 mmol) of diisopropyl ethylamine and $365 \mu \mathrm{L}(4.81 \mathrm{mmol})$ of chloromethyl methyl ether. The reaction mixture was stirred at room temperature for 40 min. The reaction mixture was diluted with $\mathrm{CHCl}_{3}$, washed with $0.5 \mathrm{M}$ citric acid and dried over $\mathrm{MgSO}_{4}$. The solvents were removed with rotovap and high vacuum rotovap to give $1.065 \mathrm{~g}(3.380 \mathrm{mmol}, 85 \%$ yield $)$ of 4 .

4. ${ }^{1} \mathrm{H}$ NMR $\left(\mathrm{CDCl}_{3} / \mathrm{TMS}\right) \delta 7.70(\mathrm{~s}, 1 \mathrm{H}), 7.16(\mathrm{~s}, 1 \mathrm{H}), 5.32(\mathrm{~s}, 2 \mathrm{H}), 3.52(\mathrm{~s}, 3 \mathrm{H}), 6.52(\mathrm{t}, 1 \mathrm{H}, \mathrm{J}=1.3 \mathrm{~Hz}), 4.86(\mathrm{dd}, 2 \mathrm{H}, \mathrm{J}=5.6$, $1.3 \mathrm{~Hz}), 2.07(\mathrm{t}, 1 \mathrm{H}, \mathrm{J}=5.6 \mathrm{~Hz}) ;{ }^{13} \mathrm{C}$ NMR (DMSO-d 6 /TMS) $\delta 56.23(\mathrm{q}), 59.06(\mathrm{t}), 94.80(\mathrm{t}), 103.51(\mathrm{~d}), 107.49(\mathrm{~s}), 108.96(\mathrm{~d})$, 112.75 (s), 128.19 (d), 153.51 (s), 155.13 (s), 155.76 (s), 159.93 (s); IR (ATR) 3508, 1703, 1604, 1267, 1158, 1095, 1083, 1032, $1009,974,894,842 \mathrm{~cm}^{-1}$.

\section{6-Bromo-7-methoxymethoxycoumarin-4-ylmethoxycarbonyl chloride (5).}

In a glass pressure bottle (Hyperglasstor, THG-A2, TAIATSU Techno) $1.9834 \mathrm{~g}(6.684 \mathrm{mmol})$ of triphogene was placed. To this 
195.4 $\mu \mathrm{L}(0.483 \mathrm{mmol})$ of aliquat ${ }^{\mathrm{R}} 336 \mathrm{in} \mathrm{n}$-hexane $(4 \mathrm{~mL})$ was added. The mixture was stirrer at room temperature for $19 \mathrm{hr}$ to generate phosgene. To a stirred solution of $321.7 \mathrm{mg}(1.021 \mathrm{mmol})$ of 4 in toluene $(2 \mathrm{~mL})$ and THF $(3 \mathrm{~mL})$ was added the phosgene solution $(4 \mathrm{~mL})$. The reaction mixture was stirred at $0^{\circ} \mathrm{C}$ for $3.5 \mathrm{hr}$. The solvents were removed under reduced pressure. The residual solid was washed with $\mathrm{CHCl}_{3}$ and n-hexane $(1 / 1 \mathrm{v} / \mathrm{v})$ twice to give $352.0 \mathrm{mg}(0.932 \mathrm{mmol}, 91 \%$ yield $)$ of 5 .

5. ${ }^{1} \mathrm{H}$ NMR (CDCl 3 /TMS) $\delta 3.53$ (3H, s, MOM), 5.33 (2H, s, MOM), 5.42 (2H, d, J=1.5, H-4), $6.43(1 \mathrm{H}, \mathrm{s}, \mathrm{H}-3), 7.19$ (1H, s, H8), $7.64(1 \mathrm{H}, \mathrm{s}, \mathrm{H}-5) ;{ }^{13} \mathrm{C}$ NMR ( $\left.\mathrm{CDCl}_{3} / \mathrm{TMS}\right) \delta$ 56.73(q), 67.08(t), 95.17(t), 104.19(d), 108.82(s), 111.77(d), 112.58(s), 127.27(s), 145.28(s), 150.62(s), 154.27(s), 156.74(s), 159.49(s); IR (ATR) 1756, 1719, 1603, 1273, 1164, $1151 \mathrm{~cm}^{-1}$.

\section{3-(6-Bromo-7-methoxymethoxycoumarin-4-ylmethoxycarbonyl)-1,2-dioctanoyl glycerol.}

To a stirred solution of $169.1 \mathrm{mg}(0.491 \mathrm{mmol})$ of 1,2-dioctanoyl glycerol $\left(\mathrm{diC}_{8}\right)$ in $\mathrm{CH}_{3} \mathrm{CN}(4 \mathrm{~mL})$ were added $115.3 \mathrm{mg}(0.944$ mmol) of 4-dimethylaminopyridine and $192.3 \mathrm{mg}(0.509 \mathrm{mmol})$ of $\mathbf{5}$. The reaction mixture was stirred at room temperature for 40 min. The solvents were removed with rotovap. Purification by flash column chromatography $\left(35 \mathrm{~g}\right.$ of $\mathrm{SiO}_{2}, \mathrm{n}$-hexane/AcOEt $=$ 35/10) gave $321.1 \mathrm{mg}(0.468 \mathrm{mmol} .95 \%$ yield $)$ of the title compound.

${ }^{1} \mathrm{H}$ NMR $\left(\mathrm{CDCl}_{3}\right) \delta 7.68(\mathrm{~s}, 1 \mathrm{H}), 7.17(\mathrm{~s}, 1 \mathrm{H}), 6.42(\mathrm{t}, 1 \mathrm{H}, \mathrm{J}=1.0 \mathrm{~Hz}), 5.32(\mathrm{~s}, 1 \mathrm{H}), 5.30(\mathrm{~m}, 1 \mathrm{H}), 5.28(\mathrm{~d}, 1 \mathrm{H}, \mathrm{J}=1.0 \mathrm{~Hz}), 4.45$ $(\mathrm{dd}, 1 \mathrm{H}, \mathrm{J}=11.9,4.0 \mathrm{~Hz}), 4.35(\mathrm{dd}, 1 \mathrm{H}, \mathrm{J}=11.5,4.6 \mathrm{~Hz}), 4.30(\mathrm{dd}, 1 \mathrm{H}, \mathrm{J}=11.5,5.6 \mathrm{~Hz}), 4.19(\mathrm{dd}, 1 \mathrm{H}, \mathrm{J}=11.9,5.6 \mathrm{~Hz}), 3.52(\mathrm{~s}$, $3 \mathrm{H}), 2.35(\mathrm{t}, 2 \mathrm{H}, \mathrm{J}=7.6 \mathrm{~Hz}), 2.33(\mathrm{t}, 2 \mathrm{H}, \mathrm{J}=7.6 \mathrm{~Hz}), 1.62(4 \mathrm{H}, \mathrm{m}), 1.28(16 \mathrm{H}, \mathrm{m}), 0.87(6 \mathrm{H}, \mathrm{m}) ;{ }^{13} \mathrm{C} \mathrm{NMR}\left(\mathrm{CDCl}_{3} / \mathrm{TMS}\right) \delta 173.2$ (s), $172.9(\mathrm{~s}), 159.8(\mathrm{~s}), 156.5(\mathrm{~s}), 154.2(\mathrm{~s}), 147.0(\mathrm{~s}), 127.4(\mathrm{~d}), 112.1(\mathrm{~s}), 111.8(\mathrm{~d}), 108.6(\mathrm{~s}), 104.1(\mathrm{~d}), 95.1(\mathrm{t}), 68.4(\mathrm{~s}), 66.6$ $(\mathrm{t}), 64.4(\mathrm{t}), 61.6(\mathrm{t}), 56.7(\mathrm{q}), 34.1(\mathrm{t}), 34.0(\mathrm{t}), 31.6(\mathrm{t}), 29.0(\mathrm{t}), 28.9(\mathrm{t}), 24.8(\mathrm{t}), 22.5(\mathrm{t}), 14.0(\mathrm{q}) ;$ IR (ATR) 1735, 1606, 1441, 1277, 1202, $1160 \mathrm{~cm}^{-1 ;} \mathrm{MS}$ (ESI) m/z $707.10\left(\mathrm{C}_{32} \mathrm{H}_{45}{ }^{79} \mathrm{BrO}_{11}+\mathrm{Na}^{+}\right), 709.90\left(\mathrm{C}_{32} \mathrm{H}_{45}{ }^{81} \mathrm{BrO}_{11}+\mathrm{Na}^{+}\right)$.

\section{3-(6-Bromo-7-hydroxy-4-ylmethoxycarbonyl)-1,2-dioctanoyl glycerol (7).}

A solution of $79.9 \mathrm{mg}(0.117 \mathrm{mmol})$ of 3-(6-Bromo-7-methoxymethoxycoumarin-4-ylmethoxycarbonyl)-1,2-dioctanoyl glycerol in trifluoroacetic acid $(2 \mathrm{~mL})$ was stirred at room temperature for $15 \mathrm{~min}$. The solvent was evaporated by rotovap and high vacuum rotovap to give $71.9 \mathrm{mg}(0.112 \mathrm{mmol} .96 \%$ yield $)$ of 7. 
7. ${ }^{1} \mathrm{H}$ NMR $\left(\mathrm{CDCl}_{3}\right) \delta 7.63(\mathrm{~s}, 1 \mathrm{H}), 7.05(\mathrm{~s}, 1 \mathrm{H}), 6.40(\mathrm{t}, 1 \mathrm{H}, \mathrm{J}=1.3 \mathrm{~Hz}), 5.33(\mathrm{~m}, 1 \mathrm{H}), 5.28(\mathrm{~d}, 2 \mathrm{H}, \mathrm{J}=1.3 \mathrm{~Hz}), 4.45(\mathrm{dd}, 1 \mathrm{H}, \mathrm{J}=$ 11.9, $4.0 \mathrm{~Hz}), 4.35(\mathrm{dd}, 1 \mathrm{H}, \mathrm{J}=11.5,4.3 \mathrm{~Hz}), 4.31(\mathrm{dd}, 1 \mathrm{H}, \mathrm{J}=11.5,5.3 \mathrm{~Hz}), 4.20(\mathrm{dd}, 1 \mathrm{H}, \mathrm{J}=11.9,5.6 \mathrm{~Hz}), 2.35(\mathrm{t}, 2 \mathrm{H}, \mathrm{J}=7.6$ Hz), $2.34(\mathrm{t}, 2 \mathrm{H}, \mathrm{J}=7.6 \mathrm{~Hz}), 1.62(\mathrm{~m}, 4 \mathrm{H}), 1.28(\mathrm{~m}, 16 \mathrm{H}), 0.87(\mathrm{~m}, 6 \mathrm{H}),{ }^{13} \mathrm{C} \mathrm{NMR}_{\left(\mathrm{CDCl}_{3} / \mathrm{TMS}\right)} \delta 173.4(\mathrm{~s}), 173.0(\mathrm{~s}), 160.2(\mathrm{~s})$, $155.9(\mathrm{~s}), 154.4(\mathrm{~s}), 154.2(\mathrm{~s}), 147.4(\mathrm{~s}), 126.8(\mathrm{~d}), 111.6(\mathrm{~s}), 111.3(\mathrm{~d}), 106.9(\mathrm{~s}), 104.4(\mathrm{~d}), 68.5(\mathrm{~s}), 66.6(\mathrm{t}), 64.4(\mathrm{t}), 61.7(\mathrm{t})$ $34.1(\mathrm{t}), 34.0(\mathrm{t}), 31.6(\mathrm{t}), 29.0(\mathrm{t}), 28.9(\mathrm{t}), 24.8(\mathrm{t}), 22.6(\mathrm{t}), 14.0(\mathrm{q})$; IR (ATR) 3375 (broad), 1724, 1607, 1441, 1409, 1280, $1218,1160 \mathrm{~cm}^{-1 ;} \mathrm{MS}(\mathrm{ESI}) \mathrm{m} / \mathrm{z} 663.00\left(\mathrm{C}_{30} \mathrm{H}_{41}{ }^{79} \mathrm{BrO}_{10}+\mathrm{Na}^{+}\right), 664.80\left(\mathrm{C}_{30} \mathrm{H}_{41}{ }^{81} \mathrm{BrO}_{10}+\mathrm{Na}^{+}\right)$.

\section{$O$-(6-Bromo-7-methoxymethoxycoumarin-4-ylmethoxycarbonyl)- $\mathrm{N}$-tert-butoxycarbonyl-L-tyrosine methyl ester.}

To a stirred solution of $236.8 \mathrm{mg}(0.802 \mathrm{mmol})$ of $\mathrm{N}$-tert-butoxycarbonyl-L-tyrosine methyl ester in $\mathrm{CH}_{3} \mathrm{CN}(6 \mathrm{~mL})$ were added $184.4 \mathrm{mg}(1.51 \mathrm{mmol})$ of 4-dimethylaminopyridine and $268.8 \mathrm{mg}(0.71 \mathrm{mmol})$ of $\mathbf{5}$. The reaction mixture was stirred at room temperature for $15 \mathrm{~min}$. The solvents were removed with rotovap. Purification by flash column chromatography $\left(30 \mathrm{~g}\right.$ of $\mathrm{SiO}_{2}$, $\mathrm{CH}_{2} \mathrm{Cl}_{2} / \mathrm{CH}_{3} \mathrm{OH}=100 / 1$ then $\left.\mathrm{CH}_{2} \mathrm{Cl}_{2} / \mathrm{CH}_{3} \mathrm{OH}=80 / 1\right)$ gave $455.1 \mathrm{mg}(0.71 \mathrm{mmol} .100 \%$ yield $)$ of the title compound.

${ }^{1} \mathrm{H}$ NMR $\left(\mathrm{CDCl}_{3}\right) \delta 7.72(\mathrm{~s}, 1 \mathrm{H}), 7.207 .12(\mathrm{~m}, 5 \mathrm{H}), 6.47(\mathrm{~s}, 1 \mathrm{H}), 5.38(\mathrm{~s}, 2 \mathrm{H}), 5.32(\mathrm{~s}, 2 \mathrm{H}), 5.17(\mathrm{~d}, 1 \mathrm{H}, \mathrm{J}=7.9 \mathrm{~Hz}), 4.59(\mathrm{~m}$ 1H), $3.72(\mathrm{~s}, 3 \mathrm{H}), 3.52(\mathrm{~s}, 3 \mathrm{H}), 3.15(\mathrm{dd}, 1 \mathrm{H}, \mathrm{J}=13.9,5.6 \mathrm{~Hz}), 3.04(\mathrm{dd}, 1 \mathrm{H}, \mathrm{J}=13.9,6.3 \mathrm{~Hz}), 1.42(\mathrm{~s}, 9 \mathrm{H}) ;{ }^{13} \mathrm{C} \mathrm{NMR}$ $\left(\mathrm{CDCl}_{3} / \mathrm{TMS}\right) \delta 171.9(\mathrm{~s}), 159.6(\mathrm{~s}), 156.2(\mathrm{~s}), 154.8(\mathrm{~s}), 154.0(\mathrm{~s}), 152.7$ (s), $149.6(\mathrm{~s}), 146.9$ (s), $134.3(\mathrm{~s}), 130.3$ (d), 127.3 (d), $120.6(d), 111.9(\mathrm{~s}), 111.6(\mathrm{~d}), 108.4(\mathrm{~s}), 103.8(\mathrm{~d}), 94.9(\mathrm{t}), 79.7(\mathrm{~s}), 64.5(\mathrm{t}), 56.5(\mathrm{q}), 54.1(\mathrm{~d}), 52.1(\mathrm{q}), 37.4(\mathrm{t}), 28.0(\mathrm{q})$; IR (ATR) $1735, \quad 1605, \quad 1507, \quad 1366, \quad 1243, \quad 1218,1156 \quad \mathrm{~cm}^{-1 ;} \quad \mathrm{MS} \quad$ (ESI) $\mathrm{m} / \mathrm{z} 657.95 \quad\left(\mathrm{C}_{28} \mathrm{H}_{30}{ }^{79} \mathrm{BrNO}_{11}+\mathrm{Na}^{+}\right), \quad 659.75$ $\left(\mathrm{C}_{28} \mathrm{H}_{30}{ }^{81} \mathrm{BrNO}_{11}+\mathrm{Na}^{+}\right)$.

\section{$O$-(6-Bromo-7-hydroxycoumarin-4-ylmethoxycarbonyl)-L-tyrosine methyl ester hydrochloride (8).}

Hydrogen chloride gas was introduced into a stirred solution of $62.6 \mathrm{mg} \quad(0.098 \mathrm{mmol})$ of $O$-(6-Bromo-7methoxymethoxycoumarin-4-ylmethoxycarbonyl)- $N$-tert-butoxycarbonyl-L-tyrosine methyl ester in $\mathrm{CH}_{2} \mathrm{Cl}_{2}(4 \mathrm{~mL})$ at room tremperature for $2 \mathrm{~min}$. The reaction mixture was stirred at room temperature for $3 \mathrm{hr}$. The solvent and the excess $\mathrm{HCl}$ were removed by rotovap and high vacuum rotovap to give $52.0 \mathrm{mg}(0.098 \mathrm{mmol} 100 \%$ yield $)$ of $\mathbf{8}$. 
8 (HCI salt). ${ }^{1} \mathrm{H}$ NMR $\left(\mathrm{CDCl}_{3}\right) \delta 7.83(\mathrm{~s}, 1 \mathrm{H}), 7.36-7.25(\mathrm{~m}, 4 \mathrm{H}), 6.84(\mathrm{~s}, 1 \mathrm{H}), 6.32(\mathrm{~s}, 1 \mathrm{H}), 5.47(\mathrm{~s}, 2 \mathrm{H}), 4.36(\mathrm{dd}, 1 \mathrm{H}, \mathrm{J}=7.3$, $6.3 \mathrm{~Hz}), 3.82(\mathrm{~s}, 3 \mathrm{H}), 3.31(\mathrm{dd}, 1 \mathrm{H}, \mathrm{J}=14.2,6.3 \mathrm{~Hz}), 3.20(\mathrm{dd}, 1 \mathrm{H}, \mathrm{J}=14.2,7.3 \mathrm{~Hz}) ;{ }^{13} \mathrm{C} \mathrm{NMR}\left(\mathrm{CDCl}_{3} / \mathrm{TMS}\right) \delta 170.3(\mathrm{~s}), 162.3$ (s), $159.3(\mathrm{~s}), 155.7$ (s), 154.5 (s), 152.2 (s), 150.3 (s), 133.7 (s), 131.8 (d), 129.5 (d), 122.8 (d), 112.0 (s), 110.8 (d), 108.1 (s), 104.4 (d), 66.4 (t), 55.1 (q), 53.7 (d), 36.7 (t); IR (ATR) 3383, 1175, 1736, 1608, 14006, 1272, 1249, $1235 \mathrm{~cm}^{-1 ;} \mathrm{MS}$ (ESI) m/z $491.90\left(\mathrm{C}_{21} \mathrm{H}_{18}{ }^{79} \mathrm{BrNO}_{8}+\mathrm{H}^{+}\right), 493.75\left(\mathrm{C}_{21} \mathrm{H}_{18}{ }^{81} \mathrm{BrNO}_{8}+\mathrm{H}^{+}\right)$.

\section{5'-(6-Bromo-7-methoxymethoxycoumarin-4-ylmethoxycarbonyl)-2',3'-O-isopropylideneadenosine.}

To a stirred suspension of $248.4 \mathrm{mg}(0.757 \mathrm{mmol})$ of 4 in $\mathrm{CH}_{3} \mathrm{CN}(6 \mathrm{~mL})$ were added $194.8 \mathrm{mg}(1.60 \mathrm{mmol})$ of 4 dimethylaminopyridine and $167.5 \mathrm{mg}(0.814 \mathrm{mmol})$ of 4-nitrophenyl chloroformate at room temperature. After $1.5 \mathrm{hr}$, another $110.3 \mathrm{mg}(0.903 \mathrm{mmol})$ of 4-dimethylaminopyridine and $253.9 \mathrm{mg}(0.826 \mathrm{mmol})$ of 2',3'-isopropylideneadenosine were added. The reaction mixture was stirred at room temperature for $3.5 \mathrm{hr}$. The solvents were removed with rotovap. The residue was diluted with $\mathrm{CHCl}_{3}(30 \mathrm{~mL})$, and washed with $0.5 \mathrm{M}$ citric acid and sat. $\mathrm{NaCl}$. The organic layer was dried over $\mathrm{MgSO}_{4}$, and the solvent was removed by rotovap and high vacuum rotovap. Purification by flash column chromatography $\left(35 \mathrm{~g}\right.$ of $\mathrm{SiO}_{2}, \mathrm{CH}_{2} \mathrm{Cl}_{2} / \mathrm{CH}_{3} \mathrm{OH}=$ 30/1 then $\left.\mathrm{CH}_{2} \mathrm{Cl}_{2} / \mathrm{CH}_{3} \mathrm{OH}=21 / 1\right)$ gave $307.4 \mathrm{mg}(0.474 \mathrm{mmol}$. $63 \%$ yield $)$ of the product.

${ }^{1} \mathrm{H}$ NMR $\left(\mathrm{CDCl}_{3} / \mathrm{TMS}\right) \delta 1.42(\mathrm{~s}, 3 \mathrm{H}), 1.63(\mathrm{~s}, 3 \mathrm{H}), 3.51(\mathrm{~s}, 3 \mathrm{H}), 4.41(\mathrm{dd}, 1 \mathrm{H}, \mathrm{J}=11.2,5.9 \mathrm{~Hz}), 4.51(\mathrm{dd}, 1 \mathrm{H}, \mathrm{J}=11.2,4.0 \mathrm{~Hz})$, $4.56(\mathrm{~m}, 1 \mathrm{H}), 5.16(\mathrm{dd}, 1 \mathrm{H}, \mathrm{J}=6.3,3.0 \mathrm{~Hz}), 5.22(\mathrm{~d}, 2 \mathrm{H}, \mathrm{J}=1.7 \mathrm{~Hz}), 5.31(\mathrm{~s}, 2 \mathrm{H}), 5.51(\mathrm{dd}, 1 \mathrm{H}, \mathrm{J}=6.3,2.0 \mathrm{~Hz}), 6.19(\mathrm{~d}, 1 \mathrm{H}, \mathrm{J}=$ $2.0 \mathrm{~Hz}), 6.31(\mathrm{br}, 2 \mathrm{H}), 6.44(\mathrm{t}, 1 \mathrm{H}, \mathrm{J}=1.7 \mathrm{~Hz}), 7.12(\mathrm{~s}, 1 \mathrm{H}), 7.60(\mathrm{~s}, 1 \mathrm{H}), 7.94(\mathrm{~s}, 1 \mathrm{H}), 8.32(\mathrm{~s}, 1 \mathrm{H}) ;{ }^{13} \mathrm{C} \mathrm{NMR}\left(\mathrm{CDCl}_{3} / \mathrm{TMS}\right)$ $\delta 25.3(q), 27.0(q), 56.6(q), 64.2(\mathrm{t}), 68.1(\mathrm{t}), 81.4(\mathrm{~d}), 84.3(\mathrm{~d}), 84.8$ (d), 90.7 (d), $95.0(\mathrm{t}), 103.9(\mathrm{~d}), 108.5(\mathrm{~s}), 111.5(\mathrm{~d}), 112.0$ (s), $114.5(\mathrm{~s}), 120.0(\mathrm{~s}), 127.2$ (d), 139.5 (d), 147.0 (s), 149.1 (s), 153.1 (d), 153.9 (s), $154.0(\mathrm{~s}), 155.8$ (s), 156.3 (s), 159.9 (s); IR (ATR) 1731, 1637, 1604, 1266, 1214, $1155 \mathrm{~cm}^{-1}$; MS (ESI) $\mathrm{m} / \mathrm{z} 647.95\left(\mathrm{C}_{26} \mathrm{H}_{26}{ }^{79} \mathrm{BrN}_{5} \mathrm{O}_{10}+\mathrm{H}^{+}\right), 649.75\left(\mathrm{C}_{26} \mathrm{H}_{26}{ }^{81} \mathrm{BrN}_{5} \mathrm{O}_{10}+\mathrm{H}^{+}\right)$.

\section{5'-(6-Bromo-7-hydroxy-4-ylmethoxycarbonyl)adenosine (9).}

To a stirred solution of $181.9 \mathrm{mg}(0.281 \mathrm{mmol})$ of 5'-(6-Bromo-7-methoxymethoxycoumarin-4-ylmethoxycarbonyl)-2',3'-Oisopropylideneadenosine in trifluoroacetic acid $(2 \mathrm{~mL})$ was added $80 \mu \mathrm{L}$ of water at room temperature. After $20 \mathrm{hr}$, the solvents were evaporated by rotovap and high vacuum rotovap. Purification by flash column chromatography (15 g of $\mathrm{SiO}_{2}$, 
$\mathrm{CH}_{2} \mathrm{Cl}_{2} / \mathrm{CH}_{3} \mathrm{OH}=15 / 1$ then $\left.\mathrm{CH}_{3} \mathrm{OH}\right)$ gave $92.5 \mathrm{mg}(0.117 \mathrm{mmol}$. $42 \%$ yield $)$ of $9 \cdot 2 \mathrm{TFA}$.

9.(2TFA salt). ${ }^{1} \mathrm{H}$ NMR $\left(\mathrm{CDCl}_{3} / \mathrm{TMS}\right) \delta 8.31(\mathrm{~s}, 1 \mathrm{H}), 8.15(\mathrm{~s}, 1 \mathrm{H}), 7.84(\mathrm{~s}, 1 \mathrm{H}), 7.29(\mathrm{~s}, 2 \mathrm{H}), 6.81(\mathrm{~s}, 1 \mathrm{H}), 6.14(\mathrm{~s}, 1 \mathrm{H}), 5.93(\mathrm{~d}$, 1H, J = 4.9 Hz), $5.60(\mathrm{~d}, 1 \mathrm{H}, \mathrm{J}=5.6 \mathrm{~Hz}), 5.44(\mathrm{~d}, 1 \mathrm{H}, \mathrm{J}=5.3 \mathrm{~Hz}), 5.38(\mathrm{~s}, 2 \mathrm{H}), 4.67(\mathrm{~m}, 1 \mathrm{H}), 4.47(\mathrm{dd}, 1 \mathrm{H}, \mathrm{J}=11.2,3.6 \mathrm{~Hz}), 4.38$ (dd, 1H, J = 11.2, $6.2 \mathrm{~Hz}), 4.26(\mathrm{~m}, 1 \mathrm{H}), 4.14(\mathrm{~m}, 1 \mathrm{H}) ;{ }^{13} \mathrm{C} \mathrm{NMR}\left(\mathrm{CDCl}_{3} / \mathrm{TMS}\right) \delta 159.8(\mathrm{~s}), 156.1$ (s), $154.2(\mathrm{~s}), 153.9(\mathrm{~s}), 152.7$ (d), 152.4 (s), 149.4 (s), 149.2 (s), 139.7 (d), 128.2 (d), 119.2 (s), 109.3 (s), 108.3 (d), 107.3 (s), 103.4 (d), 87.8 (d), 81.3 (d), 72.9 (d), 70.3 (d), $68.2(\mathrm{t}), 64.6$ (t); IR (ATR) 3337 (broad), 3184 (broad), 1685, 1646, 1604, 1406, 1271, 1249, 1206, $1139 \mathrm{~cm}^{-1}$; MS (ESI) $\mathrm{m} / \mathrm{z} 563.90\left(\mathrm{C}_{21} \mathrm{H}_{18}{ }^{79} \mathrm{BrN}_{5} \mathrm{O}_{9}+\mathrm{H}^{+}\right), 565.75\left(\mathrm{C}_{21} \mathrm{H}_{18}{ }^{81} \mathrm{BrN}_{5} \mathrm{O}_{9}+\mathrm{H}^{+}\right)$. 


\section{Quantum efficiency measurement.}

Into a pyrex test tube of $12 \mathrm{~mm}$ diameter was placed $2 \mathrm{~mL}$ of $10 \mu \mathrm{M}$ substrate solution in K-MOPS solution (pH 7.2) containing

$1 \%$ DMSO. The solution was irradiated at $350 \mathrm{~nm}$ using either two or four RPR $350 \mathrm{~nm}$ lamps. Aliquots of $10 \mu \mathrm{L}$ were removed periodically and analyzed by HPLC. The light output for the quantum efficiencies measurement was performed using ferrioxalate actinometry.

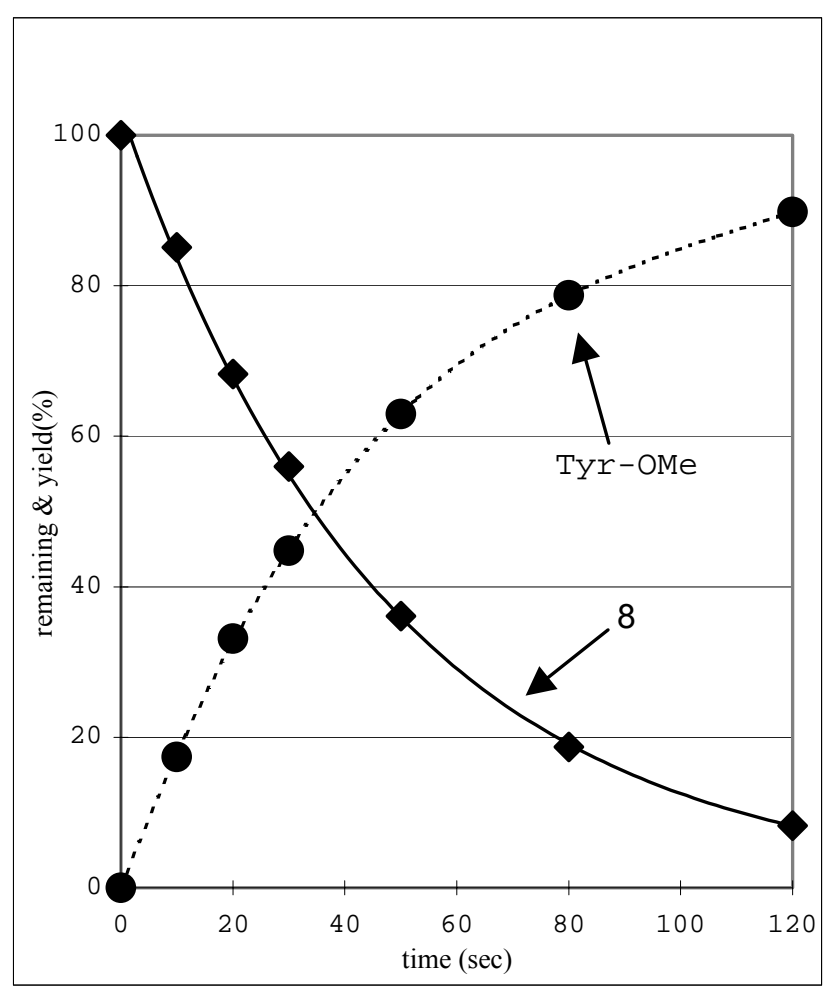

Figure 1. Time course of photolysis of Bhcmoc-TyrOMe ( 8) in solvent A upon $350 \mathrm{~nm}$ irradiation (two RPR $350 \mathrm{~nm}$ lamps). Concentrations of 8 and Tyr-OMe were quantified by HPLC analysis.

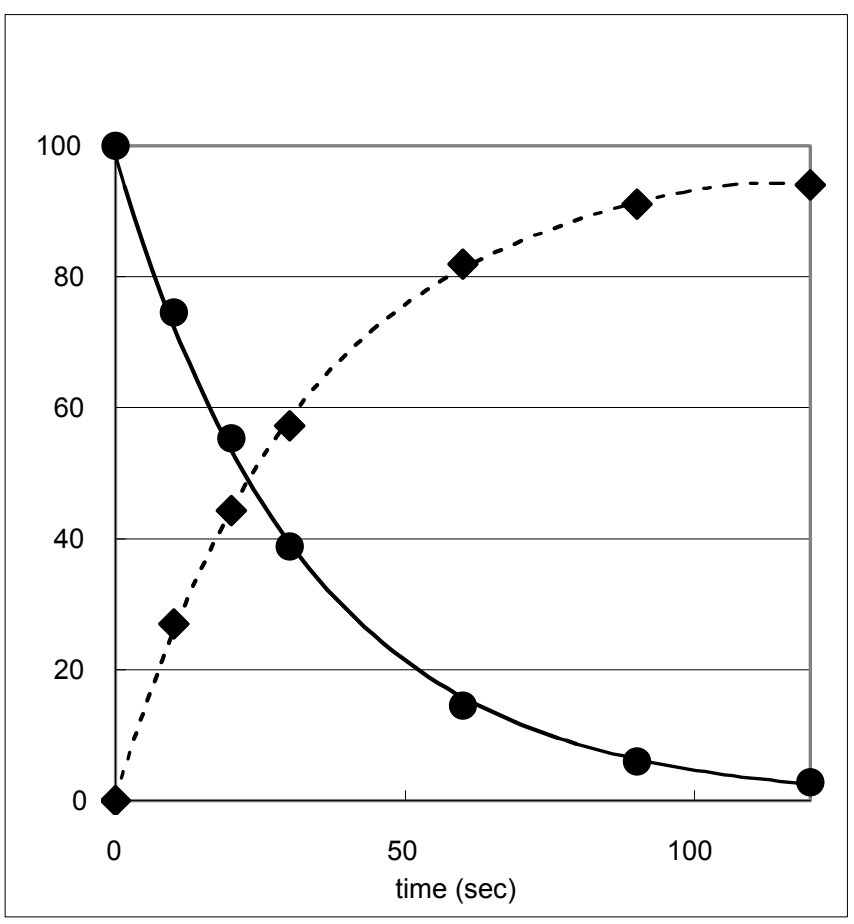

Figure 2. Time course of photolysis of 5'-Bhcmocadenosine (9) in solvent A upon $350 \mathrm{~nm}$ irradiation (four RPR $350 \mathrm{~nm}$ lamps). Concentrations of 9 and adenosine were quantified by HPLC analysis. 


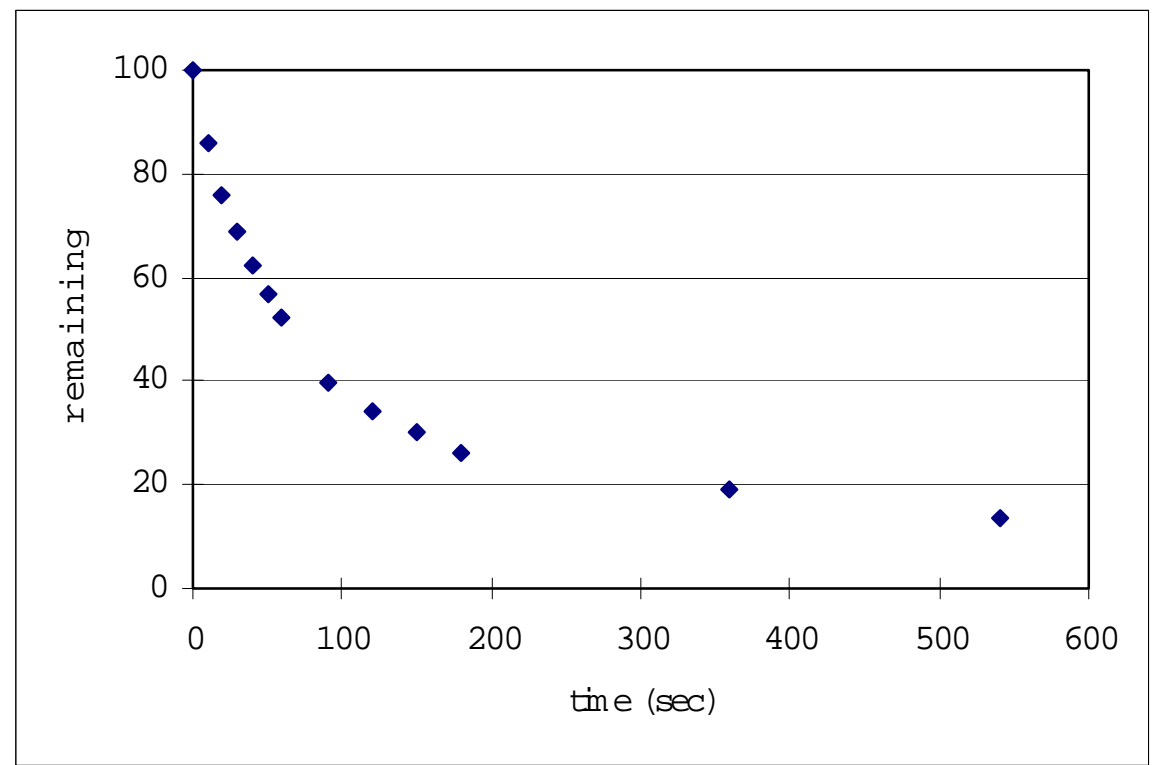

Figure 3. Time course of photolysis of Bhcmoc-dic8 (7) in solvent A upon 350 $\mathrm{nm}$ irradiation (two RPR $350 \mathrm{~nm}$ lamps). Concentrations of 7 were quantified by HPLC analysis. 